\title{
Microfluidics and Artificial Blood Vessels as Vascular Prostheses: One Small Step for Vascular Research, One Giant Leap for Patient-Kind Wendy Yang ${ }^{1 *}$ and Jianxiang Zhong ${ }^{2}$
}

${ }^{1}$ Marriotts Ridge High School, 12100 Woodford Dr, Marriottsville, MD 21104, USA

${ }^{2}$ Department of Obstetrics, Gynecology and Reproductive Sciences, University of Maryland School of Medicine, Baltimore, USA

\begin{abstract}
Studies show that in the US alone, approximately 8 million people are affected by Peripheral Vascular Disease and approximately 26.6 million have cardiovascular disease. This report will discuss the usage of lab-on-a-chip devices and artificial blood vessels as vascular prostheses and compare the strengths and weaknesses of the two methods to measure relative efficacy and possible applications in the fields of angiology and cardiology. Lab-ona-chip devices, machines which operate on a liquid scale of micro- or nano-meters and manipulate blood flow and shear stress, may be used as long-term, compact prostheses for smaller blood vessels. Artificial, man-made blood vessels, made either from cells or synthetic material, are become increasingly effective when they are made larger. Both methods may someday be instrumental in saving lives and eliminating vascular disease.
\end{abstract}

Keywords: Microfluidics; Artificial blood vessels; Vascular prostheses; Peripheral vascular disease

\section{Introduction}

According to the National Institute of Health, 1 in every 20 people over the age of 50 has Peripheral Vascular Disease in the United States. Many of these patients are in need of vascular prostheses that do not clog easily, prevent bloodstream infections, avoid triggering immune responses, are structurally sound, and can maintain patency for years on end. To find methods of treating or entirely eradicating vascular disorders, two devices that serve as alternatives for human blood vessels will be compared: the lab-on-a-chip device, a compact machine equipped with miniscule channels [1], and artificial blood vessels, which are versatile and can be grown or constructed using numerous methods [2]. Both lab-on-a-chip devices and artificial blood vessels can be used as vascular prostheses but they are made in different ways and from various materials with different functions. The lab-on-a-chip device is more effective and more efficient than artificial blood vessels in delivering fluids and nutrients to surrounding tissue and cells, while artificial blood vessels are generally more potent in providing barriers against infection and can be grown from a wide range of substances and tissues. In this review we will focus on comparing different aspects of these two methods.

\section{Lab-On-Chip Technology}

\section{Lab-on-chip device}

Lab-on-chip technology focuses on hybrid devices integrating fluidic and electronic components onto the same chip [3,4]. A typical lab-on-chip device contains microchannels, measurands, sensors, signal conditioning front-ends, analogue-to-digital converters (ADC) and a digital signal processor which analyses the signal. The analyzed data could be sent to a computer or visualized on integrated displays [1].

\section{Advantages of lab-on-chip devices}

There are many advantages of lab-on-chip devices, so they have become very attractive in many fields. Some notable advantages of lab-on- devices are compactness, automated sample handling, limited power consumption and embedded computing [1]. In addition these devices need only a minimal quantity of samples or reagents, enclosed in microchannels which could reduce the contamination of samples. Due to the small fluidic sample volumes, the lab-on-chip devices could reduce the time of analysis and measure samples while increasing precision. Moreover, these devices support a wide range of processes including sampling, transport, mixing and routing $[1,5,6]$.

\section{Applications of lab-on-chip devices}

For clinical medicine, lab-on-chip technology is applied to drug tests, glucose monitoring, diabetic control, diagnosis of disease, tests for observing pandemics and numerous other applications [1,7]. Also, lab-on-chip devices enhance biomedical research related to mixing, analysis and separation of cell suspensions, nucleic acids, proteins and so on. For example, lab-on-chip devices are being developing to perform a variety of diagnostic tests for low-resource settings, such as HIV diagnosis [8]. Rapid purification systems using antibody-based capture for subsequent diagnostic analysis also are generated with labon-chip devices [9]. Recently, a new category of lab-on-chip devices named organ-on-chip have sought to replicate in vitro organ function for drug development and specialized diagnostic applications [9]. Most importantly, lab-on-chip technology holds great promise to overcome the challenge of performing in vitro experiments in vascular research. The applications of lab-on-chip devices in vascular cell biology include the endothelial mechanorespose, migration assays, cell interactions, three-dimensional culturing, compound screening assays and stem

${ }^{*}$ Corresponding author: Wendy Yang, Marriotts Ridge High School, 12100 Woodford Dr, Marriottsville, MD 21104, Email: wyang100@gmail.com

Received June 06, 2015; Accepted December 23, 2015; Published January 02, 2016

Citation: Yang W, Zhong J (2016) Microfluidics and Artificial Blood Vessels as Vascular Prostheses: One Small Step for Vascular Research, One Giant Leap for Patient-Kind. J Biomol Res Ther 5: 135. doi:10.4172/2167-7956.1000135

Copyright: () 2016 Yang W, et al. This is an open-access article distributed under the terms of the Creative Commons Attribution License, which permits unrestricted use, distribution, and reproduction in any medium, provided the original author and source are credited. 
cells and tissue engineering.

\section{Artificial Blood Vessels}

\section{Artificial blood vessels}

Initially surgeons transplanted arteries or veins from donors to replace damaged or diseased arteries or veins, but these transplants usually failed. Even for procedures in which surgeons used vessels from patient's own body, patients would have to undergo multiple surgeries. Artificial blood vessels overcome these problems, as they are tubes made from synthetic (chemically produced) materials to restore blood circulation $[2,10]$. There are basic elements for the construction of an artificial vessel, including a structural scaffold, living cells and a nurturing environment $[11,12]$.

\section{Materials for production of artificial blood vessels}

The scaffold provides a temporary skeleton to support cell growth and molds the desired shape for the tissue. Most artificial blood vessels are based on collagen and biodegradable scaffolds, but some do not utilize structural scaffolding to this extent. Collagen scaffolding includes natural collagen, type I and type II collagen. Several biocompatible and biodegradable polymers have been used as scaffolds, such as polyglycolic acid (PGA) or PGA with polyhydroxialkanoate (PHA), poly-4-hydroxybutyrate (P4HB), poly-L-lactic acid (PLLA), and polyethylene glycol (PEG) [13]. Then smooth muscle cells, fibroblasts or endothelial cells can be seeded onto a scaffold and cultured until an optimal vessel is created. Vascular cells should be cultured in bioreactors which mimic the in vivo environment [14].

\section{Advantages of artificial blood vessels}

Nowadays artificial blood vessels are ideally composed of viable tissue. These artificial vessels are able to contract and relax, responding to forces and stimuli while secreting normal blood vessel products. Furthermore there is no any immunologic reaction of these artificial vessels, and we can remodel damaged vessels according to the needs of the environment. These vessels could grow even when transplanted into children [15].

\section{Comparison with Lab-On-A-Chip Devices and Artificial Blood Vessels}

\section{Fundamental differences}

Both lab-on-a-chip devices and artificial blood vessels can be used as vascular prostheses, though on different scales. Lab-on-a-chip (microfluidic) devices control the flow of very small quantities of fluids on a scale of micro- or nano-meters (Table 1) [16]. In contrast, artificial blood vessels tend to collapse or cause clots if they become too narrow, so they are more suitable for maintaining blood flow on a larger, visible scale, mimicking the function of major human blood vessels (Table 1) [17]. Liquids may be manipulated on both scales on the same time when artificial blood vessels are grown from microchannels in lab-ona-chip devices [18]. This can be achieved by seeding artificial blood vessels made from living tissue into premade molded channels in labon-chip devices [19]. Because of these fundamental differences, the lab-on-a-chip and artificial blood vessels have separate sets of strengths and weaknesses.

\section{Different clinical applications}

Along with lab-on-a-chip devices, artificial blood vessels, which mimic the tasks and form of human arteries and veins, can be used to restore unusable blood vessels and can treat vascular disease in vivo. Artificial blood vessels usually have several flexible layers, can be easily integrated into the human body without triggering an immune response, and should be infection-proof (Table 1) [20]. Some other vital traits found in artificial blood vessels are non-thrombogenicity, vasoactivity, and the ability to withstand sufficient burst pressure from within (Table 1) [13]. Non-thrombogenicity is the ability to prevent excessive clots in smaller blood vessels while vasoactivity is the ability to contract and relax, as valves in veins do [20]. Materials used for scaffolding act as structural support for artificial blood vessels while the use of polyurethane, a compound found in paints, forms a protective layer around the outer layer [21]. Artificial capillaries are often more difficult to replace and tend to clot more often than larger blood vessels (Table 1), so as a preventative measure, collagen scaffolding is implemented and artificial capillaries are grown from donor cells [13]. Any materials which are put into artificial blood vessels into should be screened for effects on the human body and the effects on the function of the artificial blood vessels as a whole [22]. The most commonly used synthetic materials in artificial blood vessels are Teflon and Dacron, readily available, non-toxic components which do not work well with smaller blood vessels [23]. Artificial blood vessels can be used for many purposes to advance the field of tissue engineering.

Microfluidics has many applications, several of which relate to the fields of vascularology and angiology. Microfluidics is used in cancer detection or diagnosis (Table 1), conduction of experiments on the molecular level (Table 1), and in culturing cells [16]. Lab-on-a-chip devices can manipulate the environment in which cells are cultured and regulate different aspects such as burst pressure, shear stress, and the amount of liquid being studied at a time [17]. In addition, these devices are used in the analysis of wound-healing procedures along with cell formation (Table 1) [24]. The micro channels in lab-on-a-chip devices can be used as molds to form artificial blood vessels and other types of tissue [19]. Microfluidics and artificial blood vessels may one day be implemented in the changing of lives through the construction of vascular prostheses.

\section{Different roles in biomedical research}

Both microfluidics and artificial blood vessels can be used in numerous fields in medicine. Possible future applications for artificial blood vessels include creating artificial organs and improving the process of dialysis (Table 1) [23]. Artificial blood vessels can also make way for alternate forms of treatment, like fistulas, which are hollow passages in the body. The clinical test "Vascular Access Survival and Incidence of Revisions: A Comparison of Prosthetic Grafts, Simple Autogenous Fistulas, and Venous Transposition Fistulas from the United States Renal Data System Dialysis Morbidity and Mortality Study" showed that conventional vascular prostheses like tissue grafts were less likely to be able to retain functionality after several months than fistulas would [25]. A condition such as kidney failure, which is shown to require frequent dialysis sessions, could be treated with implantable vascular grafts which purify blood in place of the kidneys [26]. There are two types of hemodialysis access: Fistula and graft [25]. In "Effectiveness of Haemodialysis Access with an Autologous Tissueengineered Vascular Graft: A Multicentre Cohort Study", vascular grafts are not likely to cause thrombosis in patients, but likely to dilate [26]. Artificial blood vessels can be used to treat various medical conditions while lab-on-a-chip devices can improve mechanical procedures to treat and modernize the diagnosis of vascular diseases (Table 1).

\section{Conclusion}


Citation: Yang W, Zhong J (2016) Microfluidics and Artificial Blood Vessels as Vascular Prostheses: One Small Step for Vascular Research, One Giant Leap for Patient-Kind. J Biomol Res Ther 5: 135. doi:10.4172/2167-7956.1000135

Page 3 of 3

\begin{tabular}{|c|c|c|}
\hline & Lab-on-a-chip devices & Artificial blood vessels \\
\hline Fundamental differences & $\begin{array}{l}\text { control the flow of very small quantities of fluids on a scale of } \\
\text { micro- or nano-meters; }\end{array}$ & $\begin{array}{l}\text { maintain blood flow on a larger and visible scale; mimic } \\
\text { the function of major human blood vessels }\end{array}$ \\
\hline Different clinical applications & $\begin{array}{l}\text { used in cancer detection or diagnosis; conduct experiments } \\
\text { on the molecular level; used in the analysis of wound-healing } \\
\text { procedures along with cell formation; used as molds to form } \\
\text { artificial blood vessels and other types of tissue; }\end{array}$ & $\begin{array}{l}\text { without triggering an immune response, and is } \\
\text { infection-proof; non-thrombogenicity, vasoactivity, } \\
\text { and the ability to withstand sufficient burst pressure; } \\
\text { is difficult to replace and tend to clot more often than } \\
\text { larger blood vessels; }\end{array}$ \\
\hline Different roles in biomedical research & $\begin{array}{l}\text { improve mechanical procedures to treat and modernize the } \\
\text { diagnosis of vascular diseases; }\end{array}$ & $\begin{array}{c}\text { creating artificial organs and improving the process of } \\
\text { dialysis; }\end{array}$ \\
\hline
\end{tabular}

Table 1: Comparison with lab-on-a-chip devices and artificial blood vessels.

Through comparison, it has been found that the lab-on-a-chip device is more effective and more efficient than artificial blood vessels in delivering fluids and nutrients to surrounding tissue and cells. However, artificial blood vessels are generally more potent in providing barriers against infection and can be grown from a wide range of substances and tissues. Both can be combined to improve the quality of life for those ailing from vascular disease and other debilitating conditions.

\section{References}

1. Giannitsis AT (2011) Microfabrication of biomedical lab-on-chip devices. A review.

2. Steinthorsson G, Sumpio B (1999) Clinical and biological relevance of vein cuff anastomosis [J]. Acta chirurgica Belgica 99: 282-288.

3. Li PCH (2010) Microfluidic lab-on-a-chip for chemical and biological analysis and discovery [M]. CRC press.

4. Zhang T, Chakrabarty K, Fair RB (2002) Microelectrofluidic Systems: Modeling and Simulation [M]. CRC Press.

5. Oh HJ, Kim SH, Baek JY (2006) Hydrodynamic micro-encapsulation of aqueous fluids and cells via'on the fly'photopolymerization [J]. Journal of micromechanics and microengineering 16: 285.

6. Gómez-Sjöberg R, Leyrat AA, Pirone DM (2007) Versatile, fully automated microfluidic cell culture system [J]. Analytical chemistry 79: 8557-8563.

7. Yager $P(2006)$ Microfluidic diagnostic technologies for global public health Nature 442: 412-418.

8. Chin CD (2011) Microfluidics-based diagnostics of infectious diseases in the developing world. Nature medicine 17: 1015-1019.

9. Eric KS (2014) The present and future role of microfluidics in biomedical research. Nature 507: 181-189.

10. Sapsford R N, Oakley G D, Talbot S (1981) Early and late patency of expanded polytetrafluoroethylene vascular grafts in aorta-coronary bypass [J]. The Journal of thoracic and cardiovascular surgery $81: 860-864$.

11. Weinberg CB, Bell E (1986) A blood vessel model constructed from collagen and cultured vascular cells. Science 231: $397-400$

12. Hoerstrup SP, Kadner A, Breymann C, et al. (2002) Living, autologous pulmonary artery conduits tissue engineered from human umbilical cord cells [J]. The Annals of thoracic surgery 74: 46-52.

13. Kakisis JD, et al. (2005) Artificial Blood Vessel: The Holy Grail of Periphera Vascular Surgery. J Vasc Surg 41: 349-354.

14. Hoerstrup SP, Zünd G, Sodian R (2001) Tissue engineering of small caliber vascular grafts [J]. Eur J Cardiothorac Surg 20:164-169.

15. Teebken O E, Haverich A (2002) Tissue engineering of small diameter vascular grafts [J]. European Journal of Vascular and Endovascular Surgery 23: 475-485.

16. Becker H, Gartner C (2012) Microfluidics and the Life Sciences. Sci Prog 95: 175-198.

17. Barber RW, David RE (2010) Biomimetic Design of Artificial Micro-vasculature for Tissue Engineering. John Hopkins Bloomberg School of Public Health. John Hopkins University. Altern Lab Anim 38: 67-79.

18. Zhou J, Laura EN (2012) Microfluidic Artificial "Vessels" for Dynamic Mechanical Stimulation of Mesenchymal Stem Cells. National Center for Biotechnology Information. National Center for Biotechnology Information. Integr Biol (Camb) 4: 1487-1497.

19. Lutolf MP, Hubbell JA (2005) Synthetic Biomaterials as Instructive Extracellular Microenvironments for Morphogenesis in Tissue Engineering. Review. Nature 23: 47-55.

20. Nerem RM (2000) Tissue Engineering a Blood Vessel Substitute: The Role of Biomechanics. National Center for Biotechnology Information. National Center for Biotechnology Information. Yonsei Med J 41: 735-739.

21. Michel RH (2005) Tissue-Engineered Blood Vessels: Alternative to Autologous Grafts? Arteriosclerosis, Thrombosis, and Vascular Biology. American Heart Association.

22. Anderson JM (2001) Biological Responses to Materials. University of UtahDepartment of Bioengineering. N.p 31: 81-110.

23. Artificial Blood Vessels. World of Invention (2006). Gale Student Resources in Context.

24. Van der Meer AD (2010) A Microfluidic Wound-healing Assay for Quantifying Endothelial Cell Migration. American Journal of Physiology. Am J Physiol Heart Circ Physiol 298: H719-25.

25. Gibson KD (2001) Vascular Access Survival and Incidence of Revisions: A Comparison of Prosthetic Grafts, Simple Autogenous Fistulas, and Venous Transposition Fistulas from the United States Renal Data System Dialysis Morbidity and Mortality Study. Elsevier Publishing Group Canada. Elsevier BV. J Vasc Surg 34: 694-700

26. McAllister TN (2009) Effectiveness of Haemodialysis Access with an Autologous Tissue-engineered Vascular Graft: A Multicentre Cohort Study. National Cente for Biotechnology Information. National Center for Biotechnology Information. The lancet 373: 1440-1446. 